\title{
Application of Compensation Algorithms to Control the Movement of a Robot Manipulator
}

\author{
Gennady K. Shadrin ${ }^{1}$, Darya L. Alontseva ${ }^{1}$, Assel T. Kussaiyn- \\ Murat $^{1}$, Albina T. Kadyroldina ${ }^{1}$, Olzhas B. Ospanov ${ }^{1}$, Tamás \\ Haidegger $^{2}$ \\ ${ }^{1}$ D. Serikbayev East Kazakhstan State Technical University, Protozanov street 69, \\ 070004, Ust-Kamenogorsk, Kazakhstan \\ shadrin.g.k@yandex.ru \\ 2 Antal Bejczy Center for Intelligent Robotics (IROB), EKIK, Óbuda University, \\ Bécsi út 96/b, H-1034 Budapest, Hungary \\ haidegger@irob.uni-obuda.hu
}

Abstract: This article presents an application-oriented method for the structural synthesis of control systems for multichannel linear objects. It provides a general form, based on the compensation for object dynamics and disturbances. The algorithm is based onalgebraic transformations of the mathematical model of the object and reference systems. The general procedure for the synthesis of a control algorithm is presented by the example of a SISO first-order object. Parametric robustness and correspondence of the system behavior to its own reference filters were derived. The possibility of applying this method to control non-linear objects presented in the form of "State Dependent Coefficient (SDC)" is ascertained. A simplified example is given by the synthesis of the motion control of a onelink manipulator with a drive, described by a second-order nonlinear equation. Control of a two-link manipulator represented by its linearized equation is demonstrated. The convenience of the proposed synthesis method for controlling multi-channel objects under certain simplifications allowed by the specifics of the use of objects is shown. The numerical example shows the independence of the system through individual channels, a zero static error in all the modes of operation and the correspondence of the system behavior to given dynamics.

Keywords: robot manipulator; control algorithm; multi-dimensional control system; reverse model; reference filter; structural synthesis

\section{Introduction}

The application of robots in the area of manufacturing is rapidly growing [1-3]. Robot manipulators are widely used in manufacturing processes, such as plasma cutting, plasma surface treatment in the form of plasma spraying of powder or 
wire coatings $[4,5]$. Plasma processing requires the accurate control of a number of parameters during the entire procedure, including the distance from the plasma system's nozzle to the surface of the workpiece, the nozzle movement speed and the angle between the plasma jet and the surface being treated [4]. Exceeding these parameters beyond the critical limits can lead not only to defective products, but also to an accident (e.g., a short circuit). In cases when the robot program is generated according to a given geometrical model of a processed workpiece or part, very often, deviation of the shape of the real object from the model leads to the violation of process parameters, with all its undesirable consequences. This problem is particularly acute in the case of objects with complex shape, when small relative errors of geometric parameters and object positioning may lead to large deviations regarding the distance between the tools mounted on the manipulator and the object's surface. Thus, it is necessary to solve the problem by adequately controlling the motion of a robotic arm and planning its trajectory. A number of research publications has addressed this issue recently [6-15]. A manipulator is a machine, in which the mechanism usually consists of a series of segments, jointed or sliding relative to one another, for the purpose of grasping and/or moving objects (pieces or tools) usually in several degrees of freedom [16]. Each joint (link) or element is equipped with its own drive, controlled by a microcontroller, which ensures the movement of the tool or the entire robot to any point of the workspace. The Kawasaki RS10L industrial robot (Kawasaki Heavy Industries, Ltd., Japan) (Fig. 1) can serve as a demonstrator, where rotational joints are used, but the motion of the tool in a preset direction is prescribed. Typically, performing robotic plasma cutting or plasma spraying requires moving the robot arm with the equipment installed on it along a given flat path with a relatively low speed, for example, about $50 \mathrm{~mm} / \mathrm{sec}$ for plasma spraying of coatings.

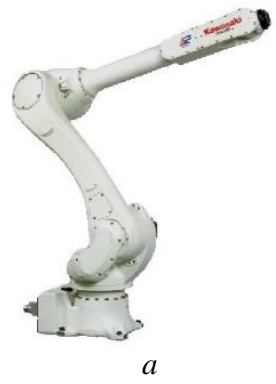

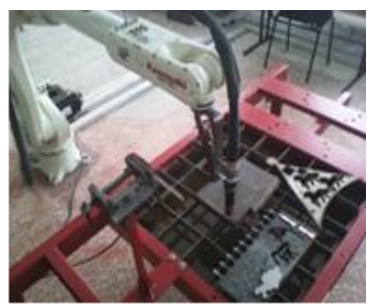

$b$

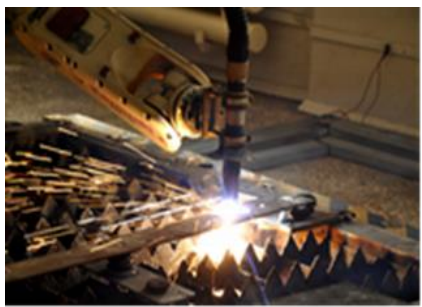

$c$

Figure 1

a) The Kawasaki RS10L industrial robot employed in the experiments; b) the robot with a plasma cutting device installed; c) a plasma cutting process in progress.

However, the constraint motion of joints or nodes, taking into account the inertia and limited power of the drives, is a rather complicated control task [8-14]. It is often required to provide the dynamics of movements' characteristic of complex robot application. 
The dynamics of the mechanical system of a manipulator is well studied, and can be described by the Lagrange equations [17-20]:

$$
\frac{d}{d t} \frac{\partial \boldsymbol{L}}{\partial \dot{\boldsymbol{q}}}-\frac{\partial \boldsymbol{L}}{\partial \boldsymbol{q}}=\boldsymbol{\tau}, L=K-P
$$

where $q \in R^{n}$ denotes the vector of generalized coordinates, $K \in R^{1}, P \in R^{1}$ the kinetic and potential energy of the manipulator, respectively, $\tau \in R^{n}$ the vector of driving moments in manipulator links and $n$ being the number of manipulator joints. After determining $\boldsymbol{L}(\boldsymbol{q}, \dot{\boldsymbol{q}})$ and calculating the derivatives, from eq. (1) for "hard" robots, the "standard" second-order vector-matrix nonlinear differential equations follow in the form:

$$
M(q) \ddot{q}+N(q, \dot{q})+G(q)=\tau,
$$

where $\boldsymbol{M}(\boldsymbol{q}) \in R^{n \times n}$ denotes the inertia matrix, $\boldsymbol{N}(\boldsymbol{q}, \dot{\boldsymbol{q}}) \in R^{n \times 1}$ the vector of Coriolis and centrifugal forces and $\boldsymbol{G}(\boldsymbol{q})$ being the gravity vector.

For a robot with rotational joints, $\boldsymbol{q}$ is the vector of rotation angles of its joints, therefore, to transform rotation angles to Cartesian coordinates, coordinate transformation should be added:

$y=f(q)$,

where $y$ denotes the vector of the Cartesian coordinates of the robot tool and $\boldsymbol{f}(\boldsymbol{q})$ being the vector function.

It should be noted that the functions included in (2), (3) are smooth nonlinear functions in the domains of their definition (trigonometric functions, squaring and certain others). In control theory, there are no universal engineering methods for the synthesis of control systems for nonlinear objects; only applications for certain classes of objects have been developed. A common technique for the design of control systems is the linearization of the object in the area of the operating point and the use of well-developed linear synthesis methods. Another approach for converting nonlinear objects to linear form in a wide range of parameter changes is feedback linearization [21, 22]; this method is widely used to synthesize control algorithms for affine systems, which includes robots, i.e., "Computed Torque Control" (CTC) [18, 22]. It should be noted the complexity of the control system obtained by CTC should be noted, given the need to calculate the second derivative of the control signal. The State Dependent Coefficient (SDC) technique [23-25] is also widespread, according to which a nonlinear object is represented as a linear model with state-dependent parameters. After linearization by any technique, the object is controlled using classical or modern linear methods of control theory. It should be noted that classical methods are poorly adapted to 
solve the problem of controlling multi-channel objects, i.e. Multiple-Input Multiple-Output (MIMO) objects.

In all cases, the task is to create a MIMO motion control system for the manipulator. Classical approaches of control algorithm synthesis are ill-posed for the task, when it includes numerous dynamic constraints, as discussed above. Most of the classical approaches are intended for the analysis of control systems for a single-channel, single-input single-output (SISO) system [26, 27]. It is necessary to analyze a number of prior defined algorithms before a given dynamic motion system is achieved. Advanced techniques, such as modal control and LQoptimization "in pure form" are means of correcting the inherent dynamics of the control object $[28,29]$. Modern deep learning techniques may also be used for deriving such complex robot controllers, if enough teaching data is available [30]. For the proper testing of external disturbances, compensators are additionally included in the system, and again, many times a trial-and-error method is used in practice. To develop an algorithm to control the robot tool motion, this article proposes a method based on the object's dynamics and perturbations compensation, offering a number of benefits [31,32]. This is an analytical method, proving the correction of the inherent dynamics of the controlled object in a single algorithm, as well as compensation for external influences, testing with zero static error. The source data is set in the form of reference filters of a closedloop system. It is easy to apply to various manipulators and tasks. The control algorithm is obtained by performing a finite number of algebraic operations over matrices. The method is based on the compensation of all external additive effects, while the precision control of the object's dynamics is achieved with referencefilters by means of inverse mathematical modeling of the object and immediately deriving the required dynamics of the system. This method has not yet been sufficiently developed and studied; there are some results for linear systems with constant parameters $[30,31]$. However, this technique may well be "adapted" for objects in the form of SDC, but this issue has yet to be considered.

The disadvantage of the method is its need for information on the state variables of the object, in case the correction of the own dynamics is required. The method has been developed for linear plants; however, research is currently being conducted on further applications for nonlinear objects in a generalized form.

Our motivation is the development of a robotic system for plasma processing of objects with complex shapes. The goal of this study is to design a new method to derive the robot manipulator's motion control. This article presents a brief description of the method of compensating the dynamics of an object and disturbances, describes the synthesis of a control algorithm using an example of a first-order object, describes the synthesis of a motion control algorithm for one link of a manipulator, when nonlinear equations of a link are presented in the form of SDC, and demonstrates the applicability of the proposed method for the synthesis of MIMO control objects using the example of a linearized model of a two-link manipulator. 


\section{Features of the Object Dynamics and Perturbations Compensation Method}

When developing the so-called "dynamics and perturbations compensation method", the control feedback is not postulated, but appears after structure's equivalent transformations obtained on the basis of the compensation method. Through this approach, it is possible to obtain a method of structural synthesis of control systems, directly guarantying the specified quality of control. The general principle of the method is to put the controlled object in series with its inverse mathematical model, and subtract all additive external disturbances from the corresponding variables of the inverse model. Disturbing effects not possible to be compensated are evaluated based on the mismatch of the corresponding object variables and the inverse model. To implement a control device in the circuit of external actions of the inverse model, the reference filters are involved, determining the behavior dynamics of a closed-loop control system. The method has an obvious advantage over the known ones regarding the clarity of the source data setting, and the simplicity of the synthesis of the control algorithm. The algorithms obtained by this method provide the compensation of disturbances with zero static error and a given dynamics changing of the controlled object. The disadvantage of the method is the need for information on the state variables of the object in case the correction of the own dynamics of this object is required. When it is not available, a state observer should be used, which is still part of our future work. A multichannel control object, represented in the state space, is considered.

$\dot{\boldsymbol{x}}_{0}^{\prime}=\boldsymbol{A} \boldsymbol{x}_{0}+\boldsymbol{B u}$,

$\boldsymbol{y}_{0}^{\prime}=\boldsymbol{C} \boldsymbol{x}_{0}$,

$\dot{x}_{0}=\dot{x}_{0}^{\prime}+f_{x}$,

$\boldsymbol{y}_{0}=\boldsymbol{y}_{0}^{\prime}+\boldsymbol{f}_{\boldsymbol{y}}$,

where $x_{0} \in \mathrm{R}^{n}$ denotes the vector of the state variables, $\boldsymbol{y}_{0} \in \mathrm{R}^{m}$ the vector of the output variables, $\boldsymbol{u} \in \mathrm{R}^{m}$ the vector of control input, $\boldsymbol{A}, \boldsymbol{B}, \boldsymbol{C}$ are the given numerical matrices of the controlled object of corresponding dimensions, $f_{y} \in \mathrm{R}^{m}$ vector denoting the perturbation added to the outcome variables and $\boldsymbol{f}_{\boldsymbol{x}} \in \mathrm{R}^{n}$ being the perturbation on the state variables. The linear system according to (4) must be kept under stable control. The method requires that the matrix composed of the first $m$ rows of the $\mathbf{B}$ matrix is a non-degenerate one. The control objective is to achieve the desired $y$ output of the system via perturbation compensation of the $f_{x}, f_{y}$ within the accuracy of the given sample dynamic systems (Fig. 2). 


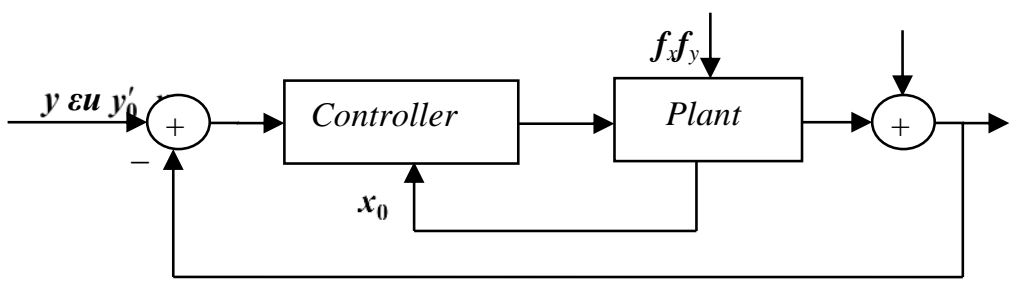

Figure 2

The block diagram of the control system

The control algorithm of the system (4) according to the method of compensation of the object's dynamics and perturbations $[31,32]$ has the form:

$\dot{x}_{\phi}=R_{1} x_{\phi}+\Phi_{2} \varepsilon$,

$\boldsymbol{u}=\boldsymbol{R}_{3} \boldsymbol{x}_{\phi}+\boldsymbol{N}_{1} \cdot \tilde{\boldsymbol{x}}_{0}+\boldsymbol{P} \boldsymbol{\varepsilon}$,

$\tilde{x}_{0}=x_{0}-x_{\phi}$,

$\boldsymbol{\varepsilon}=\boldsymbol{y}-\boldsymbol{y}_{0}$,

$\boldsymbol{R}_{1}=\Phi_{1}+\Phi_{2} \Phi_{3} ; R_{3}=\boldsymbol{N}+\boldsymbol{P} \Phi_{3} ; \boldsymbol{P}=\boldsymbol{G} \Phi_{2} ;$

$\boldsymbol{N}=\boldsymbol{G} \Phi_{1}+\boldsymbol{H} \Phi_{3} ; \boldsymbol{N}_{1}=\boldsymbol{G} \Phi_{\boldsymbol{r} 1}+\boldsymbol{H} \Phi_{\boldsymbol{r} 3}$

where $x_{\phi} \in \mathrm{R}^{n}$ is the vector of variables of regulator state; $\boldsymbol{y} \in \mathrm{R}^{m}$ being the control vector. Input variables to be controlled are $y_{0}, x_{0} ; \boldsymbol{R}_{1}, \boldsymbol{R}_{2}, \boldsymbol{R}_{3}, N, N_{1}$ are intermediate matrices.

Block matrices $\boldsymbol{E}, \boldsymbol{F}, \boldsymbol{G}, \boldsymbol{H}$ are matrices of the inverse model of the controlled system. Matrices $\boldsymbol{\Phi}_{1}, \boldsymbol{\Phi}_{2}, \boldsymbol{\Phi}_{3}, \boldsymbol{\Phi}_{\boldsymbol{r} \mathbf{1}}, \boldsymbol{\Phi}_{\boldsymbol{r} 3}$ determine sample dynamic systems, referred to as reference filters hereafter. A more detailed consideration of reference filters will be presented below. Matrices $\boldsymbol{E}, \boldsymbol{F}, \boldsymbol{G}, \boldsymbol{H}, \boldsymbol{\Phi}_{1}, \Phi_{2}, \Phi_{3}, \Phi_{\boldsymbol{r} 1}$ and $\boldsymbol{\Phi}_{\boldsymbol{r} 3}$ are interconnected with $\boldsymbol{A}, \boldsymbol{B}, \boldsymbol{C}$ by the ratio:

$$
\begin{aligned}
& {\left[\begin{array}{ll}
\boldsymbol{E} & \boldsymbol{F} \\
\boldsymbol{G} & \boldsymbol{H}
\end{array}\right]=\left[\begin{array}{ll}
\boldsymbol{A} & \boldsymbol{B} \\
\boldsymbol{C} & \mathbf{0}
\end{array}\right]^{-1},} \\
& \left\{\begin{array}{l}
\boldsymbol{E} \boldsymbol{\Phi}_{1}+\boldsymbol{F} \boldsymbol{\Phi}_{3}=\mathbf{1}, \\
\boldsymbol{E} \boldsymbol{\Phi}_{2}=\mathbf{0}, \\
\boldsymbol{\Phi}_{3} \boldsymbol{\Phi}_{1}^{-1} \boldsymbol{\Phi}_{2}=-\mathbf{1}, \\
\boldsymbol{E} \boldsymbol{\Phi}_{\boldsymbol{r} 1}+\boldsymbol{F} \boldsymbol{\Phi}_{\boldsymbol{r} 3}=\mathbf{1} .
\end{array}\right.
\end{aligned}
$$

In eqs. (7), (8) and further on in matrix expressions, $\mathbf{0}$ denotes the zero matrix, and 1 the unit (or identity) matrix with corresponding dimensions. Eq. (7) 
unambiguously determines the matrices $\mathbf{E}, \mathbf{F}, \mathbf{G}, \mathbf{H}$. If the block matrix on the right-hand side of (7) is degenerate, then the system (4) is considered uncontrolled in output and our method of synthesizing a controller shall not be used. On the contrary, ratios (8) allow for the choice of the coefficients of the reference filters. Setting these coefficients is usually intuitive when synthesizing a control algorithm particularly for a specific task for the system. The effect of the coefficients free choice, in general, has not yet been studied.

The dynamic properties of the obtained closed-loop control system are represented by the equations:

$\dot{x}_{\phi}=\Phi_{1} x_{\phi}-\Phi_{2} C \tilde{x}_{0}+\Phi_{2}\left(y-f_{y}\right)$,

$\dot{\tilde{x}}_{0}=\Phi_{r 1} \tilde{x}_{0}+f_{x}$,

$\varepsilon=-\Phi_{3} x_{\phi}-C \tilde{x}_{0}+\left(y-f_{y}\right)$,

$u=N x_{\phi}+\left(N_{1}-P C\right) \tilde{x}_{0}+P\left(y-f_{y}\right)$,

$\boldsymbol{\varepsilon}=\boldsymbol{y}-\boldsymbol{y}_{0}, \boldsymbol{P}=\boldsymbol{G} \Phi_{2}$

From these expressions it is easy to get the transfer function for:

$$
\begin{aligned}
& \varepsilon(s)=\left(\Phi_{3}\left(s \cdot 1-\Phi_{1}\right)^{-1} \Phi_{2}-1\right) C\left(s \cdot 1-\Phi_{r 1}\right)^{-1} f_{x}(s)- \\
& -\left(\Phi_{3}\left(s \cdot 1-\Phi_{1}\right)^{-1} \Phi_{2}-1\right)\left(y(s)-f_{y}(s)\right)
\end{aligned}
$$

where $s$ is the scalar complex variable of the Laplace transform. Note that in static mode $\boldsymbol{\varepsilon}(\mathbf{0})=\mathbf{0}$. This follows from (9), if we consider that according to (8) $\Phi_{3} \Phi_{1}^{-1} \Phi_{2}=-1$. Thus, the static control error is zero. From (10), it can be seen that the reference filter with matrices $\Phi_{1}, \Phi_{2}, \Phi_{3}$ determine the behavior of the closedloop system through the channels of setting job $\boldsymbol{y}$ and compensation $\boldsymbol{f}_{y}$ and is an mchannel low-pass filter.

A reference filter with the $\boldsymbol{\Phi}_{\boldsymbol{r} 1}, \boldsymbol{\Phi}_{\boldsymbol{r} 3}$ matrices can also be viewed as an $n$-channel low-pass filters through the $\boldsymbol{f}_{x}$ compensation channels. The $\boldsymbol{f}_{x}$ filter, according to (9), is connected in series with the $\boldsymbol{y}$ and $\boldsymbol{f}_{\boldsymbol{y}}$ filter.

By specifying the free coefficients of the reference filters, one can determine the frequency bandwidths of the closed-loop system and cross-links between its control channels. According to [32], for an $m$-channel controlled object with $n$ state variables, the coefficients of $m \times n$ blocks shall be free (i.e., $n$ pieces for each of the $m$ control channels) of the type:

$$
\left[\begin{array}{ccc}
\phi_{11} & \cdots & \phi_{1 n} \\
\cdots & \cdots & \cdots \\
\phi_{m 1} & \cdots & \phi_{m n}
\end{array}\right] ;\left[\begin{array}{ccc}
\phi_{r 11} & \cdots & \phi_{r 1 n} \\
\cdots & \cdots & \cdots \\
\phi_{r m 1} & \cdots & \phi_{r m n}
\end{array}\right] \text {. }
$$


The blocks in eq. (11) occupy the $\boldsymbol{\Phi}_{1}$ and $\boldsymbol{\Phi}_{\boldsymbol{r} 1}$ top rows, provided that the matrix composed of the first $m$ rows of the matrix $\mathbf{B}$ is non-degenerate. For SISO control systems, it is advisable to choose the free coefficients so that they correspond to the standard forms of the low-pass filter. As for MIMO systems, the issues of selecting the free coefficients and determining the remaining coefficients of reference filters, in general, have not yet been worked out. These tasks are being solved by considering the object properties and system requirements, as well as by solving the system (8) in each individual case. From the second equation (5), it follows that along with the main feedback in the control system, there is a local feedback on the state variables of the plant with the $N_{I}$ matrix. This feedback adjusts the dynamic properties of the object in accordance with the $f x$ reference filter. If the correction is not needed, then the parameters of this filter should be chosen, so that $N_{l}$ equals zero. Then, there is no need to measure or evaluate the state variables of the control object. Partial correction of the object is possible. Thus, according to the given mathematical model of the control plant and the specified parameters of the reference filters, the algorithm of the control device is calculated, which immediately guarantees the specified quality of the control processes. Therefore, it is of interest to consider the synthesis of algorithms and the analysis of the properties of the obtained systems specific to robot manipulators.

According to (5)-(8) by this technique, the control algorithm is obtained as a result of algebraic transformations over the matrices of the mathematical model of the control object and the matrices of filter standards. These transformations remain valid when replacing the matrix coefficients with functions of the state variables of this object, which makes it possible to use the proposed method for nonlinear objects presented in the form of SDC. Consequently, if the matrices of the filter-standard depend on state variables, then (10) is not applicable.

\section{Control Algorithm Elaboration for the Object of the First Order}

The single-channel Linear Time-Invariant (LTI)control object is represented as follows

$$
\begin{aligned}
& \dot{x}_{0}^{\prime}=a x_{0}+b u, \quad \dot{x}_{0}=\dot{x}_{0}^{\prime}+f_{x}, \\
& y_{0}^{\prime}=c x_{0}, \quad y_{0}=y_{0}^{\prime}+f_{y} \text {, }
\end{aligned}
$$

where $a, b, c$ are coefficients. The objective is to reproduce the reference signal $y$ at the output of the object and to compensate the $f_{x}, f_{y}$ perturbations within an accuracy of the given reference dynamic system. This example is handy because the structure of system (12) replicates the structure of (4), but all the block matrices in (4) are replaced by coefficients. Therefore, the solution of this 
problem, in general, is of a simple form, and at the same time allows us to follow the synthesis of the control algorithm, as well as to reveal the general properties of the obtained control system.

The synthesis of the control algorithm is performed in the following order. By (7) we calculate:

$$
\left[\begin{array}{ll}
\boldsymbol{E} & \boldsymbol{F} \\
\boldsymbol{G} & \boldsymbol{H}
\end{array}\right]=\left[\begin{array}{ll}
e & f \\
g & h
\end{array}\right]=\left[\begin{array}{ll}
a & b \\
c & 0
\end{array}\right]^{-1}=\left[\begin{array}{cc}
0 & 1 / c \\
1 / b & -a / c b
\end{array}\right],
$$

where $e, f, g, h$ are coefficients of the inverse model of the object (4). To determine the coefficients of reference filters, we record the equations (8):

$\frac{1}{c} \phi_{3}=1, \phi_{3} \frac{1}{\phi_{1}} \phi_{2}=-1, \frac{1}{c} \phi_{r 3}=1$.

According to (14), we have three equations to determine the five coefficients of the reference filters $\phi_{1}, \phi_{2}, \phi_{3}, \phi_{r 1}, \phi_{r 3}$, hence, two coefficients from among them can be set arbitrarily. Considering (11), we assign as free $\phi_{1}$ and $\phi_{r 1}$. Solving (14), we get the matrices of reference filters:

$$
\left[\begin{array}{cc}
\boldsymbol{\Phi}_{1} & \boldsymbol{\Phi}_{2} \\
\boldsymbol{\Phi}_{3} & \mathbf{0}
\end{array}\right]=\left[\begin{array}{cc}
\phi_{1} & \phi_{2} \\
\phi_{3} & 0
\end{array}\right]=\left[\begin{array}{cc}
\phi_{1} & -\frac{\phi_{1}}{c} \\
c & 0
\end{array}\right],\left[\begin{array}{c}
\boldsymbol{\Phi}_{r 1} \\
\boldsymbol{\Phi}_{r 3}
\end{array}\right]=\left[\begin{array}{c}
\phi_{r 1} \\
\phi_{r 3}
\end{array}\right]=\left[\begin{array}{c}
\phi_{r 1} \\
c
\end{array}\right] .
$$

Substituting the coefficients from (14), (15) into (6), we find

$$
\begin{aligned}
& r_{1}=\phi_{1}-\frac{\phi_{1} c}{c}=0, p=g \cdot f_{2}=-\frac{\phi_{1}}{b c}, n=g \cdot \phi_{1}+h \phi_{3}=\frac{\phi_{1}}{b}-\frac{a}{b}, \\
& n_{1}=g \cdot \phi_{r 1}+h \phi_{3}=\frac{\phi_{r 1}-a}{b}, r_{3}=n+p \phi_{3}=\frac{\phi_{1}}{b}-\frac{a}{b}-\frac{\phi_{1} c}{b c}=-\frac{a}{b} .
\end{aligned}
$$

Substituting (16) into (5), we record the object control algorithm (12):

$$
\begin{aligned}
& \dot{x}_{\phi}=-\frac{\phi_{1}}{c} \varepsilon, \quad \tilde{x}_{0}=x_{0}-x_{\phi}, \quad \varepsilon=y-y_{0}, \\
& u=-\frac{a}{b} x_{\phi}+\frac{\phi_{r 1}-a}{b} \tilde{x}_{0}-\frac{\phi_{1}}{b c} \varepsilon .
\end{aligned}
$$

Thus, a proportional-integral (PI) control law is obtained with local feedback through the variable $\tilde{x}_{0}=x_{0}-x_{\phi}$.

Processes in a closed loop control system consisting of the object (12) and the regulator (17) after substitution (13) and (15) in (9) take the form: 
$\dot{x}_{\phi}=\phi_{1} x_{\phi}+\phi_{1} \tilde{x}_{0}-\frac{\phi_{1}}{c}\left(y-f_{y}\right)$,

$\dot{\tilde{x}}_{0}=\phi_{r 1} \tilde{x}_{0}+f_{x}$,

$\varepsilon=-c x_{\phi}-c \tilde{x}_{0}+\left(y-f_{y}\right)$,

$u=\frac{\phi_{1}-a}{b} x_{\phi}+\frac{\phi_{r 1}-a+\phi_{1}}{b} \tilde{x}_{0}-\frac{\phi_{1}}{c}\left(y-f_{y}\right)$.

As seen from (18), the free coefficients of the reference filter $\phi_{1}$ and $\phi_{r 1}$ determine the dynamic properties of the control system. For the sustainability of the processes in (18), they must be negative. The study of the influence of reference filters coefficients on the control process was performed by computer-based simulation method and was about to analyze the transients in a system composed of (12) and (17) for a numerical example when $a=-1, b=c=1$. Figure 3 shows the transient response of the system for the values $\phi_{1}=-0.5$ (blue), $\phi_{1}=-1$ (red) and $\phi_{1}=-1.5$ (brown). In Fig. 3and other figures, at zero time, the input was changed according to a unit step, after 10 seconds, a single step perturbation $f_{y}$, appeared, and after 20 seconds appeared a single step perturbation $f_{x}$ of a negative sign.

As seen, the approach to increase the system performance by the increase of $\phi_{1}$ leads to significant amplitude brightening of the control input. Apparently, it is advisable for this example when $\phi_{1}=1$, then the amplitude of the control action is moderate and characterized by a high speed of operation. Fig. 4 shows transition functions similar to Figure 3 , but here the value of $\phi_{r 1}$ changed when $\phi_{1}=1$. Expectedly from (18), this parameter affects only the nature of compensation of the perturbation of $f_{x}$. As seen in Fig. 4, it is possible to increase $\phi_{r 1}$ without increasing the amplitude of the control input.

a

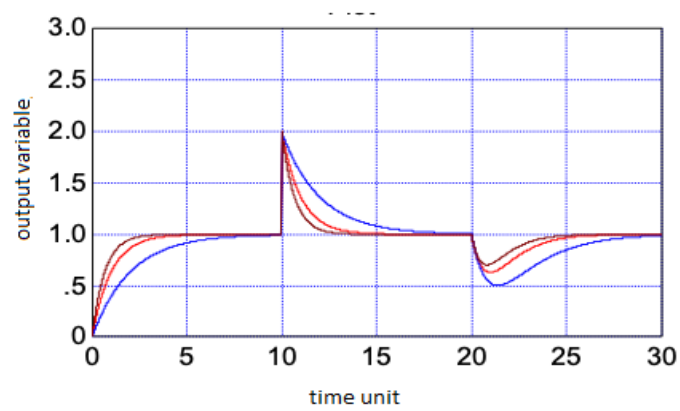


b

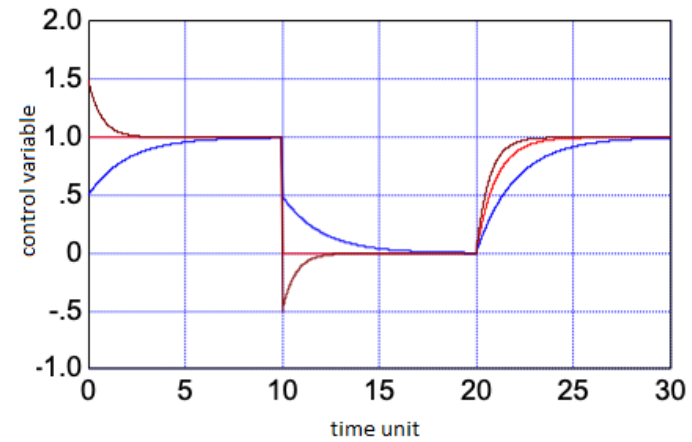

Figure 3

Input signal to test the system (12), with controller (17) when changing $\phi_{1}$ : a) output variable, b) control variable

a

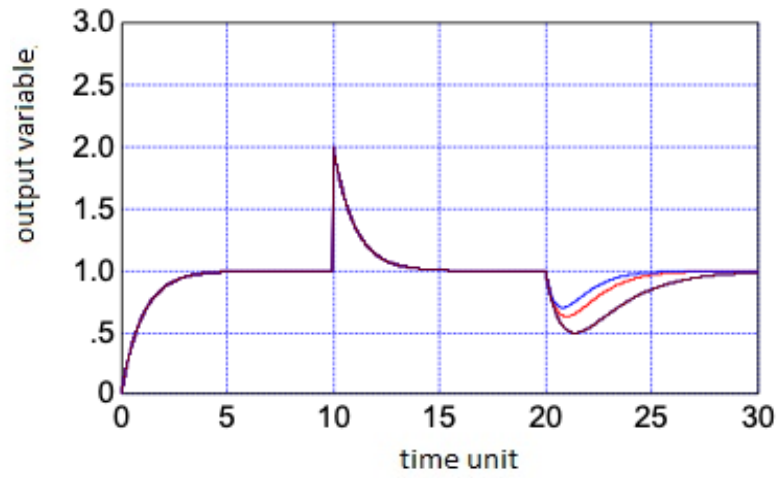

b

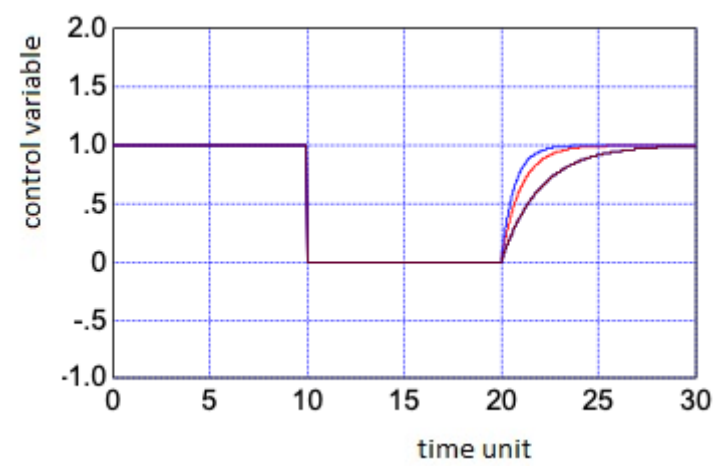

Figure 4

Input signal to test the system (12), with controller (17) when changing $\phi_{r 1}$ a) output variable, b) control variable 
The study of robustness for a given set of coefficients was to analyze transition functions while a significant change of the coefficients of the control object and the regulator being calculated for the nominal values of these coefficients. Figure 5 demonstrates these functions. Here, the coefficients $a, b, c$ of the system (12) were successively changed by $50 \%$ from their nominal values.

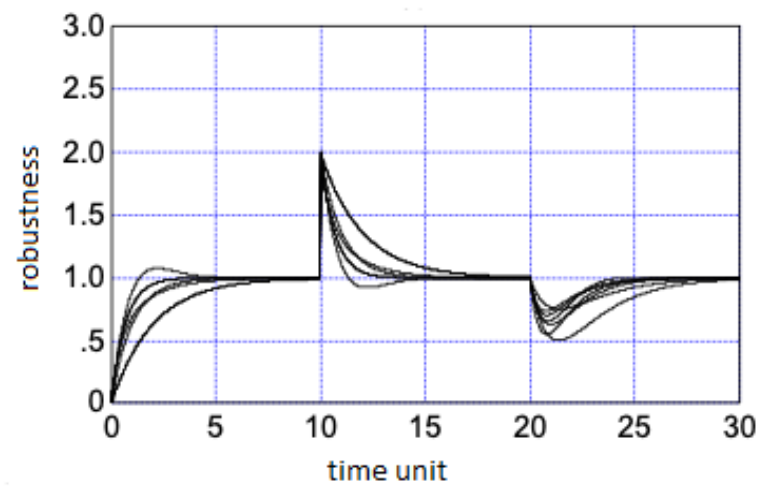

Figure 5

The study of the control system robustness (12), (17)

As seen, the system remains stable and the control quality is adequate. Simulation with a number of other sets of object coefficients (12), including for an unstable object, revealed similar results.

\section{Motion Control of One Manipulator Joint}

Let us consider the algorithm synthesis of the rotation angle control of one link of a rotary type manipulator. For simplicity, we neglect the dynamics of the electromagnetic processes of the drive and friction in the joints. In addition, we believe that the mass of the link is concentrated at its end point. Eq. (2) for one link, the axis of rotation of which is fixed at the origin, has the form:

$\left(J_{p}+m l^{2}\right) \ddot{q}+m g l \cos (q)=k u$,

where $m$ denotes the link mass, $l$-the link length, $q$ the link rotation angle relative to the horizontal axis, $g$ the acceleration of gravity and $J_{p}$ being the drive moment of inertia. It was assumed in (19) that the driving moment created by the drive is connected with the control signal to this drive $u$ by the formula $\tau=k u$, where $k$ is the proportionality coefficient. 
To synthesize a control algorithm, these equations must be represented in the state space. Dividing the right and left sides of (19) into $J_{p}+m l^{2}$ and denoting $x_{01}=\dot{q}$, $x_{02}=q, \quad a_{1}=\frac{m g l}{J_{p}+m l^{2}}, \quad b=\frac{k}{J_{p}+m l^{2}}, \quad$ where $k$ is a coefficient of proportionality, we obtain the mathematical model of the control object in the form:

$\dot{x}_{01}=-a_{1} \cos x_{02}+b u$,

$\dot{x}_{02}=x_{01}$,

$y_{0}=x_{02}$.

We represent (20) in the form of SDC; for this, we divide and multiply the first term in (20) by $x_{02}$, in the end we get:

$\dot{x}_{01}=a(\cdot) \cdot x_{02}+b u$,

$\dot{x}_{02}=x_{01}$,

$y_{0}=x_{02}$,

where

$a(\cdot)=a\left(x_{02}\right)=-\frac{a_{1}}{x_{02}} \cos x_{02}, x_{02} \neq 0$.

Now in notation (4), we have:

$\boldsymbol{A}=\left[\begin{array}{cc}0 & a(\cdot) \\ 1 & 0\end{array}\right], \boldsymbol{B}=\left[\begin{array}{l}b \\ 0\end{array}\right], \boldsymbol{C}=\left[\begin{array}{ll}0 & 1\end{array}\right]$.

We carry out the synthesis of the control algorithm according to (5)-(8) taking into account (11). By (7) we calculate:

$$
\boldsymbol{E}=\left[\begin{array}{ll}
0 & 1 \\
0 & 0
\end{array}\right], \boldsymbol{F}=\left[\begin{array}{l}
0 \\
1
\end{array}\right], \boldsymbol{G}=\left[\begin{array}{cc}
\frac{1}{b} & 0
\end{array}\right], H=-\frac{a(\cdot)}{b} .
$$

According to (8), we compose equations for determining filter standards:

$$
\begin{aligned}
& \boldsymbol{E}=\left[\begin{array}{ll}
0 & 1 \\
0 & 0
\end{array}\right], \boldsymbol{F}=\left[\begin{array}{l}
0 \\
1
\end{array}\right], \boldsymbol{G}=\left[\begin{array}{ll}
\frac{1}{b} & 0
\end{array}\right], H=-\frac{a(\cdot)}{b} \cdot \boldsymbol{E} \boldsymbol{\Phi}_{1}+\boldsymbol{F} \boldsymbol{\Phi}_{3}= \\
& {\left[\begin{array}{ll}
0 & 1 \\
0 & 0
\end{array}\right]\left[\begin{array}{ll}
\phi_{1} & \phi_{2} \\
\phi_{21} & \phi_{22}
\end{array}\right]+\left[\begin{array}{l}
0 \\
1
\end{array}\right]\left[\begin{array}{ll}
\phi_{31} & \phi_{32}
\end{array}\right]=\left[\begin{array}{ll}
1 & 0 \\
0 & 1
\end{array}\right],} \\
& \boldsymbol{E \Phi}_{2}=\left[\begin{array}{ll}
0 & 0 \\
0 & 1
\end{array}\right]\left[\begin{array}{l}
\phi_{13} \\
\phi_{23}
\end{array}\right]=\left[\begin{array}{l}
0 \\
0
\end{array}\right],
\end{aligned}
$$




$$
\begin{aligned}
& \boldsymbol{\Phi}_{\mathbf{3}} \boldsymbol{\Phi}_{\mathbf{1}}^{-\mathbf{1}} \boldsymbol{\Phi}_{\mathbf{2}}=\left[\begin{array}{ll}
\phi_{31} & \phi_{32}
\end{array}\right]\left[\begin{array}{cc}
\phi_{1} & \phi_{2} \\
\phi_{21} & \phi_{12}
\end{array}\right]^{-1}\left[\begin{array}{l}
\phi_{13} \\
\phi_{23}
\end{array}\right]=-1, \\
& \boldsymbol{E} \boldsymbol{\Phi}_{\boldsymbol{r} 1}+\boldsymbol{F} \boldsymbol{\Phi}_{\boldsymbol{r} \mathbf{3}}=\left[\begin{array}{ll}
0 & 1 \\
0 & 0
\end{array}\right]\left[\begin{array}{cc}
\phi_{r 1} & \phi_{r 2} \\
\phi_{r 21} & \phi_{r 12}
\end{array}\right]+\left[\begin{array}{l}
0 \\
1
\end{array}\right]\left[\begin{array}{ll}
\phi_{r 31} & \phi_{r 32}
\end{array}\right]=\left[\begin{array}{ll}
1 & 0 \\
0 & 1
\end{array}\right] .
\end{aligned}
$$

According to (11), we assign the following coefficients as free ones: $\phi_{1}, \phi_{2}, \phi_{r 1}$, $\phi_{r 2}$. Then, solving (25), we obtain:

$$
\boldsymbol{\Phi}_{\mathbf{1}}=\left[\begin{array}{cc}
\phi_{1} & \phi_{2} \\
1 & 0
\end{array}\right], \boldsymbol{\Phi}_{\mathbf{2}}=\left[\begin{array}{c}
-\phi_{2} \\
0
\end{array}\right], \boldsymbol{\Phi}_{\mathbf{3}}=\boldsymbol{\Phi}_{r \mathbf{3}}=\left[\begin{array}{ll}
0 & 1
\end{array}\right], \boldsymbol{\Phi}_{r \mathbf{1}}=\left[\begin{array}{cc}
\phi_{r 1} & \phi_{r 2} \\
1 & 0
\end{array}\right] .
$$

Substituting (24), (26) into (5) and (6) and taking into account (22) we obtain the object control algorithm (21):

$$
\begin{aligned}
& \dot{x}_{\phi 1}=\phi_{1} x_{\phi 1}-\phi_{2} \varepsilon \\
& \dot{x}_{\phi 2}=x_{\phi 1}, \\
& u=\frac{\phi_{1}}{b} x_{\phi 1}+\left(\frac{a_{1}}{b x_{02}} \cos x_{02}\right) x_{\phi 2}+\frac{\phi_{r 1}}{b} \tilde{x}_{01}+\frac{1}{b}\left(\phi_{r 2}+\frac{a_{1}}{x_{02}} \cos x_{02}\right) \tilde{x}_{02}-\frac{\phi_{2}}{b} \varepsilon \\
& \tilde{x}_{01}=x_{01}-x_{\phi 1}, \\
& \tilde{x}_{02}=x_{02}-x_{\phi 2} \\
& \varepsilon=y-y_{0} .
\end{aligned}
$$

Substituting (24), (26) into (9) derives equations defining the processes in a closed control system:

$$
\begin{aligned}
& \dot{x}_{\phi 1}=\phi_{1} x_{\phi 1}+\phi_{2} x_{\phi 2}+\phi_{2} \tilde{x}_{02}-\phi_{2}\left(y-f_{y}\right), \\
& \dot{x}_{\phi 2}=x_{\phi 1}, \\
& \dot{\tilde{x}}_{01}=\phi_{r 1} \tilde{x}_{01}+\phi_{r 2} \tilde{x}_{02}+f_{x 1}, \\
& \dot{\tilde{x}}_{02}=\tilde{x}_{01}+f_{x 2},
\end{aligned}
$$

As it can be seen, despite the nonlinearity of the object, processes in a closed system are represented by linear equations of filter standards. Let us consider free coefficients of reference filters. It follows from (28) that simulating the $\left(y-f_{y}\right)$ signal by a closed loop system is characterized by the transfer function of a lowpass filter of the second order:

$$
W(s)=\frac{\phi_{2}}{s^{2}+\phi_{1} s+\phi_{2}}=\frac{\omega_{\phi}^{2}}{s^{2}+\varsigma_{\phi} \omega_{\phi} s+\omega_{\phi}^{2}}, \phi_{1}=-\varsigma_{\phi} \omega_{\phi}, \phi_{2}=-\omega_{\phi}^{2},
$$


where $\omega_{\phi}$ denotes the natural frequency of the filter, $\varsigma_{\phi}$ the coefficient of damping and $s$ being the Laplace transform variable. Record in the (23) form is favorable for reference filters forms applying. For example, for the second-order Bessel filter $\varsigma_{\phi} \approx 1,73$. Changing $\omega_{\phi}$, it is possible at the given damping of $\varsigma_{\phi}$ to select the desired speed of the filter and the entire closed-loop control system. The above reasoning is also valid for the filter through $\boldsymbol{f}_{x}$ simulating channels. Computer simulation of the system (20), (27) in the case of substituting a number of coefficients (19) demonstrated the correspondence of transient processes to the equations (28)

\section{Motion Control of a Two-Link Manipulator}

Consider the problem of a given movement of the operating point of a flat twolink manipulator of rotational type. The axis of rotation of the first link is fixed relative to the coordinate system; the axis of the second link is located on the first link.

The dynamics of this manipulator can be described in the form [18]:

$$
\begin{aligned}
& {\left[\begin{array}{cc}
\alpha+\beta+2 \eta \cos q_{2} & \beta+\eta \cos q_{2} \\
\beta+\eta \cos q_{2} & \beta
\end{array}\right]\left[\begin{array}{l}
\ddot{q}_{1} \\
\ddot{q}_{2}
\end{array}\right]+\left[\begin{array}{c}
-\eta\left(2 \dot{q}_{1} \dot{q}_{2}+\dot{q}_{2}^{2}\right) \sin q_{2} \\
\eta q_{1}^{2} \sin q_{2}
\end{array}\right]+} \\
& {\left[\begin{array}{c}
\alpha e_{1} \cos q_{2}+\eta e_{1} \cos \left(q_{1}+q_{2}\right) \\
\eta e_{1} \cos \left(q_{1}+q_{2}\right)
\end{array}\right]=\left[\begin{array}{l}
k_{1} u_{1} \\
k_{2} u_{2}
\end{array}\right],} \\
& y_{01}=l_{1} \cos q_{1}+l_{2} \cos q_{2}, \\
& y_{02}=l_{1} \sin q_{1}+l_{2} \sin q_{2},
\end{aligned}
$$

where $\alpha, \beta, \eta, e_{1}, k_{1}, k_{2}, l_{1}, l_{2}$ denote coefficients depending on the parameters of links and drives and $y_{01}, y_{01}$ being Cartesian coordinates of the working point on the plane. Eq. (29) can be represented in the state space, they can be transformed exactly or approximately to the form (4) with state-dependent coefficients (SDC form), and then the control algorithm can be synthesized according to (5)-(8). But this difficult task has not yet been solved and may be the object of future research. In this section, a simpler problem was posed, namely, using the linearized model of a two-link manipulator as an example to show the ease of synthesis of control algorithms for a two-channel LTI object by the proposed technique, which immediately provides the specified indicators of control quality. This refers to the usual linearization in the area of the working point. Introducing the notation $x_{01}=\dot{q}_{1}, \quad x_{02}=\dot{q}_{2}, \quad x_{03}=q_{1}, \quad x_{04}=q_{2}$, and performing such linearization, we obtain the following equations: 
$\dot{x}_{01}=a_{11} x_{01}+a_{12} x_{02}+a_{13} x_{03}+a_{14} x_{04}+b_{1} u_{1}$,

$\dot{x}_{02}=a_{21} x_{01}+a_{22} x_{02}+a_{23} x_{03}+a_{24} x_{04}+b_{2} u_{2}$,

$\dot{x}_{03}=x_{01}$,

$\dot{x}_{04}=x_{02}$,

$y_{01}=c_{13} x_{03}+c_{14} x_{04}$,

$y_{02}=c_{23} x_{03}+c_{24} x_{04}$,

In (31), for brevity, the initial notation of variables for increments is preserved.

The general formulas being clumsy, the solution is made for a numerical illustration. Matrix for a numerical illustration we assign in the form (32):

$$
\boldsymbol{A}=\left[\begin{array}{cccc}
-5 & 0 & 0 & 0 \\
0 & -5 & 0 & 0 \\
1 & 0 & 0 & 0 \\
0 & 1 & 0 & 0
\end{array}\right], \boldsymbol{B}=\left[\begin{array}{cc}
10 & 0 \\
0 & 10 \\
0 & 0 \\
0 & 0
\end{array}\right], \boldsymbol{C}==\left[\begin{array}{cccc}
0 & 0 & -1 & -2 \\
0 & 0 & 3 & 4
\end{array}\right]
$$

We take $b_{1}=10, b_{2}=10$. The zero elements in the first two lines of A are accepted for ease of calculation. Non-zero coefficients of the matrix $\boldsymbol{A}$ in (30) provide drives of links with a sufficiently high speed. In matrix $\boldsymbol{C}$, these coefficients are assigned arbitrarily. Substituting (31) into (7) we get>

$$
\begin{aligned}
& \boldsymbol{E}=\left[\begin{array}{llll}
0 & 0 & 1 & 0 \\
0 & 0 & 0 & 1 \\
0 & 0 & 0 & 0 \\
0 & 0 & 0 & 0
\end{array}\right], \boldsymbol{F}=\left[\begin{array}{cc}
0 & 0 \\
0 & 0 \\
2 & 1 \\
-1,5 & -0,5
\end{array}\right], \\
& \boldsymbol{G}==\left[\begin{array}{cccc}
0,1 & 0 & 0,5 & 0 \\
0 & 0,1 & 0 & 0,5
\end{array}\right], \boldsymbol{H}==\left[\begin{array}{ll}
0 & 0 \\
0 & 0
\end{array}\right] .
\end{aligned}
$$

By (11) blocks of freely assigned coefficients are:

$$
\left[\begin{array}{llll}
\phi_{11} & \phi_{12} & \phi_{13} & \phi_{14} \\
\phi_{21} & \phi_{22} & \phi_{23} & \phi_{24}
\end{array}\right] ;\left[\begin{array}{llll}
\phi_{r 11} & \phi_{r 12} & \phi_{r 13} & \phi_{r 14} \\
\phi_{r 21} & \phi_{r 22} & \phi_{r 23} & \phi_{r 24}
\end{array}\right] .
$$

The analysis (9) proves that the variables $x_{\phi}$ and $\tilde{x}_{0}$ in their physical sense correspond to the state variables in (4). It follows that some of the coefficients in (34) denote interconnections between state variables. Since we have to ensure the independence of control channels of the reference system, the coefficients of interconnections should be taken as zero. As a result, we have the following blocks of assigned coefficients: 


$$
\left[\begin{array}{cccc}
\phi_{11} & 0 & \phi_{13} & 0 \\
0 & \phi_{22} & 0 & \phi_{24}
\end{array}\right] ;\left[\begin{array}{cccc}
\phi_{r 11} & 0 & \phi_{r 13} & 0 \\
0 & \phi_{r 22} & 0 & \phi_{r 24}
\end{array}\right] .
$$

Now, each pair of coefficients in any row (33) corresponds to a filter of a transfer function (23). Assigning $\omega_{\phi}=2, \omega_{r \phi}=4, \varsigma_{\phi}=\varsigma_{r \phi}=1,73$, we get for $>$

$$
\begin{aligned}
& {\left[\begin{array}{cccc}
\phi_{11} & 0 & \phi_{13} & 0 \\
0 & \phi_{22} & 0 & \phi_{24}
\end{array}\right]=\left[\begin{array}{cccc}
-3,46 & 0 & -4 & 0 \\
0 & -3,46 & 0 & -4
\end{array}\right],} \\
& {\left[\begin{array}{cccc}
\phi_{r 11} & 0 & \phi_{r 13} & 0 \\
0 & \phi_{r 22} & 0 & \phi_{r 24}
\end{array}\right]=\left[\begin{array}{cccc}
-6,92 & 0 & -16 & 0 \\
0 & -6,92 & 0 & -16
\end{array}\right] .}
\end{aligned}
$$

The remaining elements of the reference filters are calculated by (8). The resulting matrices of a reference filter are recorded as:

$$
\begin{aligned}
& \boldsymbol{\Phi}_{1}=\left[\begin{array}{cccc}
-3,46 & 0 & -4 & 0 \\
0 & -3,46 & 0 & -4 \\
1 & 0 & 0 & 0 \\
0 & 1 & 0 & 0
\end{array}\right], \boldsymbol{\Phi}_{2}=\left[\begin{array}{cc}
8 & 4 \\
-6 & -2 \\
0 & 0 \\
0 & 0
\end{array}\right], \boldsymbol{\Phi}_{3}= \\
& {\left[\begin{array}{cccc}
0 & 0 & -1 & -2 \\
0 & 0 & 3 & 4
\end{array}\right],} \\
& \boldsymbol{\Phi}_{\boldsymbol{r} \mathbf{1}}=\left[\begin{array}{cccc}
-6,92 & 0 & -16 & 0 \\
0 & -6,92 & 0 & -16 \\
1 & 0 & 0 & 0 \\
0 & 1 & 0 & 0
\end{array}\right], \boldsymbol{\Phi}_{\boldsymbol{r} 3}=\left[\begin{array}{cccc}
0 & 0 & -1 & -2 \\
0 & 0 & 3 & 4
\end{array}\right] .
\end{aligned}
$$

Solving the system of algebraic equations (8) when the given coefficient blocks are known, may be of some difficulty. Substituting (33), (37) into (5) and (6), we obtain the equations of the object control algorithm (31), (32) in the form> 
$\dot{x}_{\phi 1}=-3,46 x_{\phi 1}+8 \varepsilon_{1}+4 \varepsilon_{2}$,

$\dot{x}_{\phi 2}=-3,46 x_{\phi 2}-6 \varepsilon_{1}-2 \varepsilon_{2}$,

$\dot{x}_{\phi 3}=x_{\phi 1}$,

$\dot{x}_{\phi 4}=x_{\phi 2}$,

$u_{1}=0,154 x_{\phi 1}-0,192 \tilde{x}_{01}-1.6 \tilde{x}_{03}+0,8 \varepsilon_{1}+0,4 \varepsilon_{2}$,

$u_{2}=0,154 x_{\phi 2}-0,192 \tilde{x}_{02}-1,6 \tilde{x}_{04}-0,6 \varepsilon_{1}-0,2 \varepsilon_{2}$,

$\tilde{x}_{01}=x_{01}-x_{\phi 1}, \quad \tilde{x}_{02}=x_{02}-x_{\phi 2}$,

$\tilde{x}_{03}=x_{03}-x_{\phi 3}, \quad \tilde{x}_{04}=x_{04}-x_{\phi 4}$,

$\varepsilon_{1}=y_{1}-y_{01}, \quad \varepsilon_{2}=y_{2}-y_{02}$.

Fig. 6 depicts the transition functions obtained on a computer model of a system (31), (32), (38) where coordinates of a given position of the operating point $y_{1}, y_{2}$ were changed in a stepwise way. In one second, the reference $y_{1}$ was changed, in 5 seconds - the reference $y_{2}$ was changed.

Figs. $6 a$ and $6 b$ demonstrate the changing of the actual coordinates of $y_{01}$ and $y_{02}$, and Figures $6 c$ and $6 d$ demonstrate the changes in the control actions on the first and second drives during these tasks simulation.

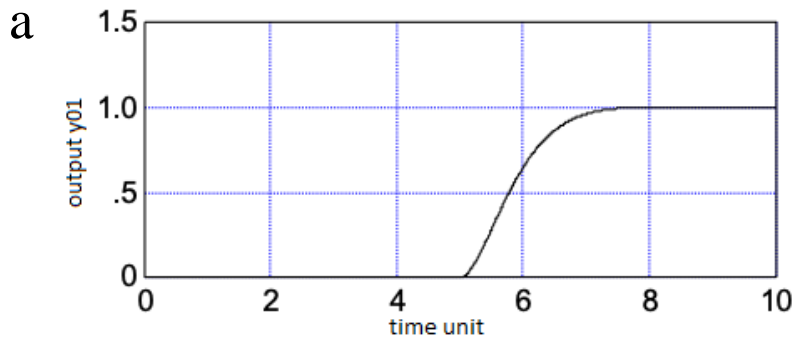

b

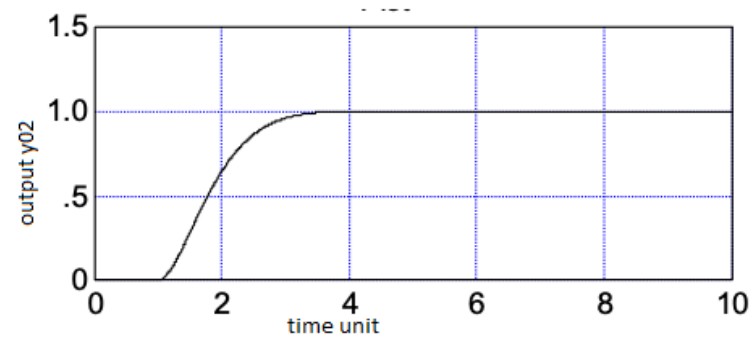



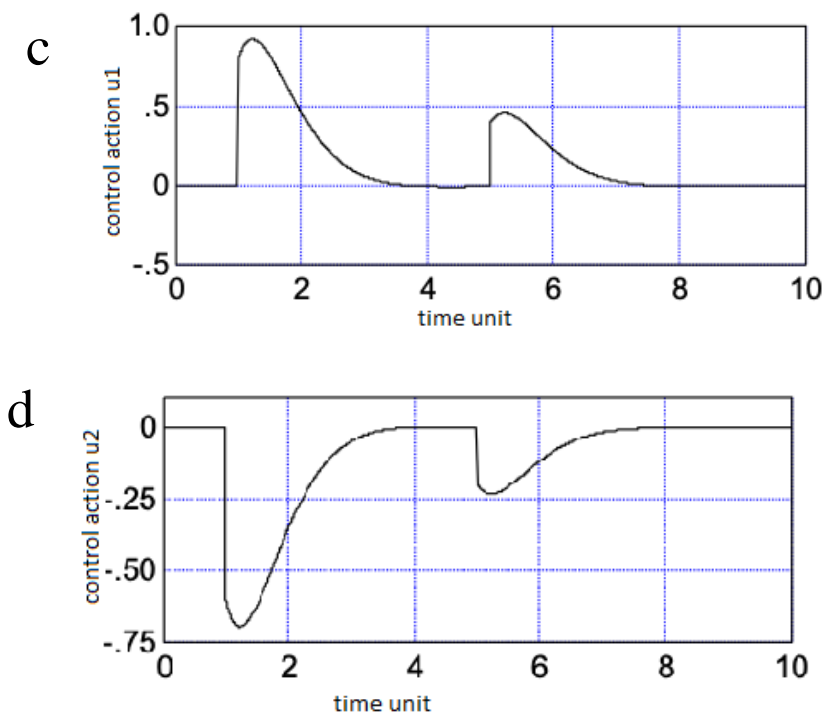

Figure 6

Simulating a unit step change of reference signals $y_{1}, y_{2}$ by the control system(31), (32), (38): a-b) changing $y_{01}$ and $y_{02}, \mathrm{c}-\mathrm{d}$ ) changing the control actions $u_{1}$ and $u_{2}$.

Fig. 7 shows the simulation by the system of unit step changes of $f_{x 1}, f_{x 2}$ perturbations. These perturbations simulate changes in the load on the drives. 
C

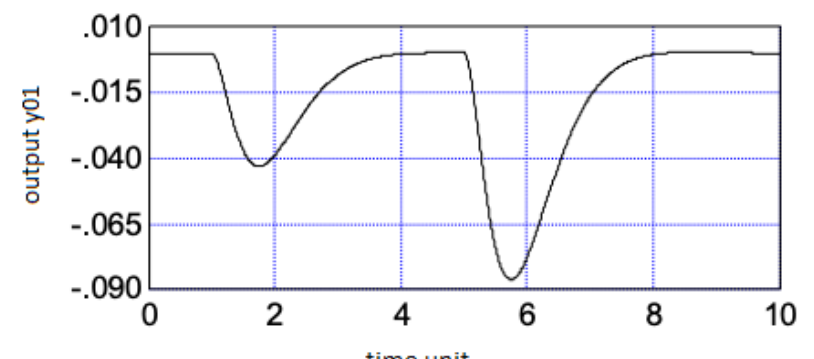

b

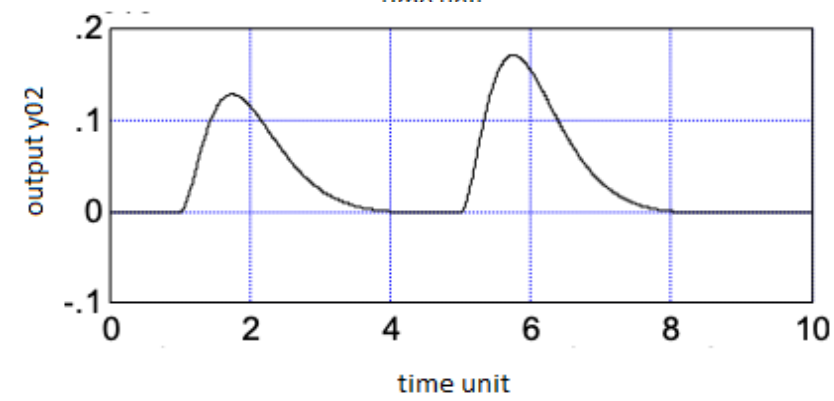

c

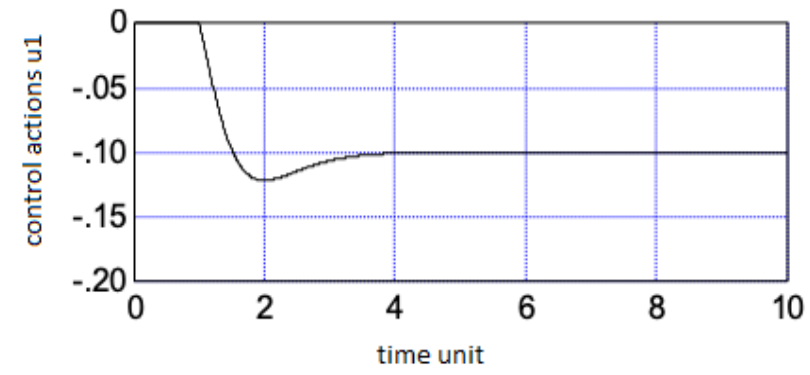

d

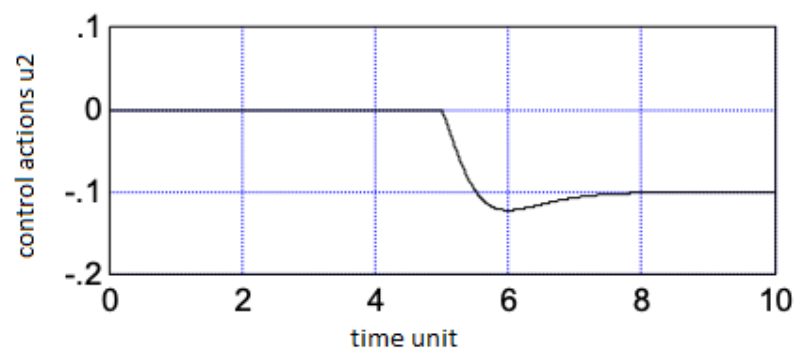

Figure 7

Simulating a unit step change of reference signals $y_{1}, y_{2}$ by the control system (31), (32), (38): a-b) changing $y_{01}$ and $y_{02}, \mathrm{c}-\mathrm{d}$ ) changing the control actions $u_{1}$ and $u_{2}$ 
Based on Figs. 6 and 7 it is seen that the complete self-regulation through channels has been achieved: references $y_{1}, y_{2}$, outputs $y_{01}, y_{02}$ and zero static control error. The control processes dynamics satisfies a given dynamics of reference filters. Obviously, autonomy is violated if the actual parameters of the control object do not match their calculated values, but the control error is always zero. This has been revealed by research methods on the computer model of the system.

Thus, a new method for compensating dynamics and perturbations of the object is considered, which allows developing the structure and parameters of a control algorithm for a multi-channel linear object. Here, the standard forms of lowfrequency filters are proposed to be used and the effectiveness of their application is demonstrated. This synthesis technique application for multichannel objects is considered on the example of a two-link manipulator represented by its linearized equation. The calculation of the regulator for a numerical example of such an object has highlighted the complexity of solving a problem of selecting the remaining coefficients of reference filters when the blocks of assigned coefficients are given. Analytical research and computer modeling of the considered examples proved the correspondence of transients in control systems to a given dynamics in case of external actions, the autonomy of the SISO systems through separate control channels, zero static error and the robustness of the obtained systems. It is revealed that these properties are achieved immediately, without applying the trial and error method. The ease of the initial data assigning for the synthesis of systems should be noted. The drawbacks of the algorithms are the need for information on the state variables or higher complexity when using state observers. Generally, the issues of free coefficients determining and techniques for non-free coefficients of reference filters identifying have not been resolved yet. Once completely developed, the controller shall be applicable to robotic application with complex geometry constraints, such as plasma processing, or even for medical applications, such as automated bone surface milling for implants [33].

\section{Conclusions}

The new method of synthesis of a robot manipulator motion-control system by dynamics and perturbations compensation has been proposed. Under certain assumption, this method allows to develop the structure and parameters of a control algorithm for a multi-channel linear object. The possibility of applying the proposed algorithm to control nonlinear objects represented in the form of SDC is demonstrated. On the example of a simple SISO system of first order, duplicating the general structure, but giving a simple analytical solution, the sequence of synthesis of the control algorithm, and the principles of choosing free coefficients of reference filters for the general case are considered. On the example of the task of controlling the rotation of a link of a manipulator, the synthesis of a control algorithm for a nonlinear object is considered and the method of selecting free coefficients for a link of the second order is described. The obtained control 
algorithm can be applied to automate a wide class of industrial facilities. The proposed method of structural synthesis can be used to develop effective motion control systems for robot manipulators. The results of the research are of significance for a range of application domains, developing control algorithms for production sites with mechatronic systems.

\section{Acknowledgement}

This work was supported by the Ministry of Education and Science of the Republic of Kazakhstan under the target financing program for the 2017-2019 years by the sub-program 0006/ PCF-17 "Manufacture of titanium products for further use in medicine". T. Haidegger is supported through the New National Excellence Program of the Hungarian Ministry of Human Capacities. T. Haidegger is a Bolyai Fellow of the Hungarian Academy of Sciences. The final grammatical improvements of the article were supported by the V4+ACARDC CLOUD AND VIRTUAL SPACES grant.

\section{References}

[1] T. Haidegger, G. S. Virk, C. Herman, R. Bostelman, P. Galambos, G. Györök, I. J. Rudas: Industrial and Medical Cyber-Physical Systems: Tackling User Requirements and Challenges in Robotics, In Recent Advances in Intelligent Engineering, Springer, Cham, 2020, pp. 253-277

[2] J. Somló, G. Dávid Varga, M. Zenkl, B. Mikó: The „Phantom” Delta Robot A New Device for Parallel Robot Investigations, Acta Polytechnica Hungarica 15, No. 4, 2018, pp. 143-160

[3] G. Haidegger, I. Paniti: Issues in Manufacturing Automation \& Robotics within the Past 4 Decades and Vision for the Next, Proceedings of IEEE International Conference on Intelligent Engineering Systems (INES), 2019, pp. 23-28

[4] R. C. Tucker, Jr., editor: Introduction to Coating Design and Processing, Thermal Spray Technology, Vol. 5A, 2013, pp. 76-88

[5] D. L. Alontseva, A. L. Krasavin, A. T. Kadyroldina, A. T. Kussaiyn-Murat, D. M. Nurekenov, Ye. T. Zhanuzakov, N. V. Prokhorenkova: Development of the Robotic Microplasma Spraying Technology for Applying Biocompatible Coatings in the Manufacture of Medical Products, Proceedings of AIS $2017-12^{\text {th }}$ International Symposium on Applied Informatics and Related Areas, Székesfehérvár, Hungary, 2017, pp. 45-48

[6] L. Márton, Z. Szántó, T. Haidegger, P. Galambos, J. Kövecses: Internetbased bilateral teleoperation using a revised time-domain passivity controller, Acta Polytechnica Hungarica, Vol. 14, No. 8, 2017, pp. 27-45

[7] E. Horvátha, C. Pozna, R.-E. Precup: Robot Coverage Path Planning Based on Iterative Structured Orientation, Acta Polytechnica Hungarica 15, No. 2, 2018, pp. 231-249 
[8] R. Zhao: Trajectory planning and control for robot manipulations. Robotics [cs.RO], thesis - Université Paul Sabatier - Toulouse III, 2015, pp. 158, URL: https://tel.archives-ouvertes.fr/tel-01285383v2/document

[9] R. Campa, C. Ramirez, K. Camarillo, V. Santibanez, I. Soto: Motion Control of Industrial Robots in Operational Space: Analysis and Experiments with the PA10 Arm, Advances in Robot Manipulators, Ernest Hall (Ed.), 2010, pp. 417-442, ISBN: 978-953-307-070-4

[10] K. M. Lynch, F. C. Park: Modern Robotics Mechanics, Planning and Control, Cambridge U. Press, 2017, pp. 642, ISBN: 978-110-715-630-2

[11] A. Nemeikšis: Trajectory modulation of 2.5 degree of freedom robot arm, Scientific researches and their practical application, Proceedings of Modern state and ways of development, Vol. 5, № 3, 2014, pp. 3-11, URL: https://elibrary.ru/item.asp?id=22270213

[12] S. Yurish, editor: Advances in Robotics and Automatic Control: Reviews, Book Series. Vol. 1, 2018, pp. 404, ISBN: 978-84-09-02449-0

[13] Zh.-G Liu., J.-M. Huang: A New Adaptive Tracking Control Approach for Uncertain Flexible Joint Robot System, Proceedings of International Journal of Automation and Computing, Vol. 12(5), 2015, pp. 559-566, DOI: $10.1007 / \mathrm{s} 11633-015-0898-6$

[14] Zh. Wang, W. Liu, B. Cui, J. He, Zh. Li, Yo. Zhao: Research on Robot Surface Tracking Motion Based on Force Control of Six-Axis Wrist Force Sensors, Advances in Mechanical Engineering, ID: 249696, 2014, pp.1-9

[15] Gy. Eigner: Control of Physiological Systems through Linear Parameter Varying Framework, Acta Polytechnica Hungarica, Vol. 14, No. 6, 2017, pp. $185-212$

[16] ISO 8373:2015 ISO. 8373:2015 robot vocabulary standards. Manipulating industrial robots

[17] M. Vukobratovic, V. Potkonjak: Scientific Fundamentals of Robotics 1, Dynamics of Manipulation Robots: Theory and Application, SpringerVerlag, Vol. 1, 1982, ISBN 978-3-642-81854-7

[18] F. L. Lewis, S. Jagannathan, A. Yesildirek: Neural Network Control of Robot Manipulators and Nonlinear Systems, Taylor \& Francis, 1999, ISBN 0-7484-0596-8

[19] B. Armstrong, O. Khatib, J. Burdick: The explicit dynamic model and internal parameters of the PUMA 560 arm, Proc. IEEE Conf. On Robotics and Automation, 1986, pp. 510-518

[20] J. Somló, B. Lantos, P. T. Cát: Advanced Robot Control, Akadémiai Kiadó, Budapest, 2002, p. 428, ISBN10 9630573504 
[21] A. Isidori: Nonlinear Control Systems, Berlin: Springer, 1995, pp. 549, DOI 10.1007/978-1-84628-615-5

[22] N. K. Khalil: Nonlinear Systems, $3^{\text {rd }}$ ed. New Jersey: Prentice Hall, 2002, pp. 575, DOI: 10.3969/j.issn.1006-7043. 201112013

[23] B. Friedland: Quasi Optimal Control and the SDRE Method, Proc. 17'IFAC Symp. on Automatic Control in Aerospace. Toulouse, France, 2007

[24] S. Skogestad, I. Postlethwaite: Multivariable Feedback Control, $2^{\text {nd }}$ ed. Wiley-Interscience, 2005, p. 592, ISBN: 0470011688, 9780470011683

[25] J. S. Shamma, J. R. Cloutier:Existence of SDRE Stabilizing Feedback, IEEE Transactions on Automatic Control, No. 6 (3), 2003, pp. 513-517 DOI: 10.1109/TAC.2002.808473

[26] C. Dorf Richard, H. Bishop Robert: Modern Control Systems, $12^{\text {th }}$ ed.Prentice Hall, 2011, p. 1111

[27] A. G. Aleksandrov: K analiticheskomu sintezu regulyatorov [To analytical synthesis of regulators], Automatics and telemechanics, No. 6, 2010, pp. 319 (in Russian)

[28] A. M. Letov: Analiticheskoe konstruirovanie regulyatorov I, II, III [Analytical design of regulators I, II, III], Automatics and telemechanics, No. 4, 1960, pp. 406-411; No. 5, 1960, pp. 561-568; No. 6, 1960, pp. 661665 (in Russian)

[29] R. Isermann: Digital Control Systems, Springer Science \& Business Media, 2013, p. 566, ISBN 3662023199, 978366202319

[30] A. I. Károly, R. Fullér, P. Galambos: Unsupervised Clustering for Deep Learning: A tutorial survey, Acta Polytechnica Hungarica, Vol. 15, No. 8, 2018, pp. 29-53

[31] G. K. Shadrin: A Physics-Based Approach to Control Systems Design Using Compensation of Controlled Plant Dynamics and Perturbations, Proceedings of Automation and Remote Control, Vol. 77, No. 7, 2016, pp. $1151-1162$

[32] G. K. Shadrin, D. A. Porubov, M. G. Shadrin: Sintez algoritma upravleniya dvizheniem dvuhkolesnogo robota metodom kompensacii dinamiki ob"ekta i vozmushchenij [Synthesis of two-wheeled robot motion control algorithm by compensating object dynamics and disturbances], Proceedings of Automatics \& software enginery, No. 4 (22), 2017, pp. 10-17 (in Russian)

[33] T. Haidegger: Autonomy for Surgical Robots: Concepts and Paradigms, Proceedings of IEEE Transactions on Medical Robotics and Bionics 1, No. 2, 2019, pp. 65-76, DOI 10.1109/TMRB.2019.2913282 